\title{
Extruded sorghum (Sorghum bicolor L.) reduces metabolic risk of hepatic steatosis in obese rats consuming a high fat diet
}

\author{
Andressa Rodrigues de Sousa ${ }^{a}$, Maria Eliza de Castro Moreira ${ }^{a}$, Renata Celi Lopes Toledo ${ }^{a}$, \\ Laércio dos Anjos Benjamin $^{\mathrm{b}}$, Valéria Aparecida Vieira Queiroz ${ }^{\mathrm{c}}$, Marcia Paranho Veloso ${ }^{\mathrm{d}}$, \\ Kassius de Souza Reis ${ }^{\mathrm{d}}$, Hércia Stampini Duarte Martino ${ }^{\mathrm{a}, *}$ \\ ${ }^{a}$ Department of Nutrition and Health, Federal University of Vicosa, MG, Brazil \\ b Department of Veterinary, Federal University of Viçosa, MG, Brazil

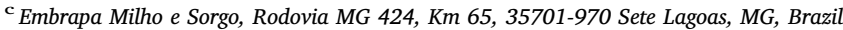 \\ ${ }^{\mathrm{d}}$ Faculty of Pharmaceutical Sciences, Federal University of Alfenas, MG, Brazil
}

\section{A R T I C L E I N F O}

\section{Keywords:}

Sorghum

Adipogenese

Anthocyanin

Bioactive compounds

$\beta$-Oxidation

Tannins

\begin{abstract}
A B S T R A C T
The study investigated the effect of extruded sorghum flour (ESF) in a high fat diet (HFD) on biometric measurements and hepatic lipogenesis. Male Wistar rats were fed a normal diet (AIN-93M), HFD, HFD plus ESF replacing 50\% cellulose and 100\% corn starch (HFDS50), or HFD plus ESF replacing 100\% cellulose and 100\% corn starch (HFDS100) for eight weeks. ESF reduced the body mass index and liver weight of obese rats. Additionally, ESF reduced hepatic lipogenesis by increasing adiponectin 2 receptor gene expression and gene and protein expressions of peroxisome proliferator-activated receptor $\alpha$ (PPAR $\alpha$ ), while reducing the gene expression of sterol regulatory element-binding transcription factor 1. Molecular docking analysis revealed the affinity of ESF compounds (luteolinidin, apigeninidin, 5-methoxy-luteolinidin, and 7-methoxy-apigeninidin) with the PPAR- $\alpha$ receptor. Histological analysis confirmed the decreased grade of hepatic steatosis in obese rats. These data indicate the potential of ESF to reduce metabolic risk of hepatic steatosis associated with lipogenesis and obesity.
\end{abstract}

\section{Introduction}

An imbalance between energy intake and energy expenditure contributes to the development of obesity. Consumption of a hypercaloric diet contributes to the accumulation of visceral fat and lipotoxicity in tissues, including the liver (Lyons, Kennedy, \& Roche, 2016). Lipotoxicity activates inflammatory pathways, which promotes increased oxidative stress, deregulated lipid metabolism, and induction of dyslipidemia and hepatic steatosis, which lead to pathologies such as nonalcoholic fatty liver disease (NAFLD) (Ceriello \& Motz, 2004).

NAFLD is one of the most prevalent chronic liver diseases. It is recognized as a hepatic manifestation from metabolic syndrome and is characterized by simple steatosis, which can lead to non-alcoholic steatohepatitis (NASH) and fibrosis and even lethal cirrhosis (Dowman, Tomlinson, \& Newsome, 2010; Szczepaniak et al., 2005). The mechanisms that lead to excessive fat deposition in the liver (steatosis) are not yet fully understood. However, according to the "two hits" hypothesis, the "first hit" is insulin resistance, which leads to lipid metabolism disorders by increasing the expression of lipogenic genes and reduced mitochondrial $\beta$-oxidation (Yamazaki et al., 2007). The "second hit" is a progression to NAFLD due to increased oxidative stress, lipid peroxidation, and activity of pro-inflammatory cytokines (Day \& James, 1998).

As the number of obese individuals and their associated diseases continues to increase, nutritional strategies aiming to prevent and treat obesity have been the focus of many studies (Belobrajdic et al., 2011; Goto et al., 2011). Sorghum (Sorghum bicolor L.), a cereal native to Africa and a member of the Poaceae family, may have nutritional and functional value of potential benefit to human health (Lopes et al., 2018; Stefoska-Needham et al., 2015). Among the sorghum genotypes, grains with the pericarp and pigmented forehead have a high content of phenolic acids, 3-deoxyanthocyanidins (3-DXA), and proanthocyanidins (tannins) (Cardoso et al., 2015). These compounds are effective in the modulation of diseases such as cancer, obesity, diabetes,

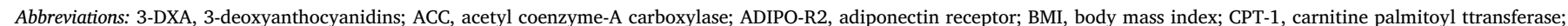

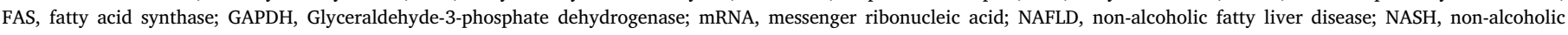
steatohepatitis; PPAR, peroxisome proliferator activated receptor; SCD1, estearoil coenzyme A desaturase 1; TNF- $\alpha$, tumor necrosis factor $\alpha$

* Corresponding author at: Departamento de Nutrição e Saúde, Universidade Federal de Viçosa, Avenida P.H. Rolfs, Campus Universitário S/N, Viçosa, MG, Brazil.

E-mail address: hercia@ufv.br (H.S.D. Martino). 
cardiovascular diseases, and others (Cardoso et al., 2017; Lopes et al., 2018). Chung et al. (2011) reported that oral ingestion of extracts of sorghum phenolic compounds ( 100 to $250 \mathrm{mg} / \mathrm{kg}$ for 14 days) is effective in reducing plasma cholesterol concentration and triacylglycerol in rats, and recent studies by our research group found that extruded sorghum flour can effectively reduce lipogenesis by reduced fatty acid synthase gene expression, improve sensitivity to insulin and reduce inflammatory cytokines, including tumor necrosis factor alpha (Arbex et al., 2018).

In order to realize the potential benefits of sorghum consumption in humans and animals, several processing of appealing and edible food products is imperative. Extrusion is a promising processing technique in functional cereal production. It is characterized by the combination of heat, moisture, and mechanical modification of raw materials to yield new shapes and structures with differing characteristics in a short period of time (Alam et al., 2016; Anunciação et al., 2017). It has been proposed that the high temperatures used in sorghum extrusion contribute to a reduction in the degree of polymerization of tannins and to fragmentation of the arabinoxylan structure, increasing their bioavailability and therefore their potential for biological effects on markers of health (Cardoso et al., 2015; Lopez et al., 2016).

Recent studies by our research group have found that extruded Sorghum has both anti-obesogenic effect and anti-inflammatory effects in obese Wistar rats treated with a high fat diet (Arbex et al., 2018). Our hypothesis is that he high level of bioactive compounds including 3DXA, proanthocyanins (tannins), and dietary fiber in brown pericarp sorghum (SC 319) (Cardoso et al., 2017) can promote hepatic lipogenesis control by blocking the production of lipogenic enzymes and increasing $\beta$-oxidation in the liver of animals fed an HFD. Then, the aim of this study was to evaluate the effects of an extruded sorghum meal (ESF) on the biometric profile and on the modulation of regulatory pathways related to lipolysis and hepatic lipogenesis.

\section{Material and methods}

\subsection{Extruded Sorghum flour}

Sorghum genotype SC 319 with brown pericarp, was selected for its high content of dietary fiber and bioactive compounds, including proanthocyanins (tannins) and 3-DXA (Cardoso et al., 2015, 2017). Sorghum genotype seeds SC 319 were planted on the Embrapa Milho and Sorgo research station, located at Nova Porteirinha, Minas Gerais, Brazil $\left(15^{\circ} 47^{\prime} \mathrm{S}\right.$ latitude coordinates; $43^{\circ} 18^{\prime} \mathrm{O}$ longitude coordinates and $516 \mathrm{~m}$ above sea level), on June 2011. Grains were then sent to Cereal Laboratory (Embrapa Agroindústria de Alimentos, Rio de Janeiro, Brazil) and were extruded, according to methods described by VargasSolorzano et al. (2014). The extruded grains were ground into flour and then packed in polyethylene bags and stored at $-20 \pm 1{ }^{\circ} \mathrm{C}$.

ESF chemical composition data, including carbohydrates, proteins, lipids, dietary fiber and bioactive compounds were previously determined by our research group (Arbex et al., 2018) and the data was used to inform the design of experimental diets in this study (Table 1).

\subsection{Animals and diets}

Wistar rats (Rattus novergicus) adult males (60 days old; $\mathrm{n}=32$ ) from the Federal University of Viçosa (UFV) were housed in individual stainless steel cages and maintained in a controlled environment with temperature of $22^{\circ} \mathrm{C} \pm 3{ }^{\circ} \mathrm{C}$, with a light-dark cycle of $12 \mathrm{~h}$ and access to water ad libitum. All experiments were conducted according to the Declaration of Helsinki on the welfare of experimental animals $\left(\mathrm{N}^{\circ} 06\right.$ ) 2014).

Initially, the rats were divided into two groups and received the following diets: high fat diet (HFD) $(n=24)$ as obese control, based on the composition of commercial diet D12079B (Research Diets ${ }^{\circledR}$ ) for obesity induction and AIN-93 M diet (Reeves, Nielsen, \& Fahey, 1993)
Table 1

Composition of the experimental diets $\left(\mathrm{g} \cdot 100 \mathrm{~g}^{-1}\right)$.

\begin{tabular}{|c|c|c|c|c|}
\hline Ingredients & AIN-93M ${ }^{\mathrm{A}}$ & $\mathrm{HFD}^{\mathrm{B}}$ & HFDS50 ${ }^{\mathrm{C}}$ & HFDS100 ${ }^{\mathrm{D}}$ \\
\hline Casein & 14 & 19.50 & 17.67 & 15.84 \\
\hline Maltodextrin & 15.50 & 10 & 3.10 & 0 \\
\hline Corn starch & 46.57 & 5.32 & 0 & 0 \\
\hline Sucrose & 10 & 34.10 & 34.10 & 25.04 \\
\hline Soybean oil & 4 & 1 & 0.66 & 0.32 \\
\hline Lard & 0 & 20 & 20 & 20 \\
\hline Cellulose & 5 & 5 & 2.5 & 0 \\
\hline Vitamin mix & 1 & 1 & 1 & 1 \\
\hline Mineral mix & 3.5 & 3.5 & 3.5 & 3.5 \\
\hline Bitartrate choline & 0.25 & 0.25 & 0.25 & 0.25 \\
\hline L-cystine & 0.18 & 0.18 & 0.18 & 0.18 \\
\hline Cholesterol & 0 & 0.15 & 0.15 & 0.15 \\
\hline BHT & 0.0008 & 0.000448 & 0.0004 & 0.0004 \\
\hline Extruded sorghum flour & - & - & 16.86 & 33.72 \\
\hline Total 3- DXAS $(\mu \mathrm{g})$ & nd & nd & 16.92 & 33.85 \\
\hline Luteolinidin $(\mu \mathrm{g})$ & nd & nd & 4.45 & 8.90 \\
\hline Apigeninidin $(\mu \mathrm{g})$ & nd & nd & 2.39 & 4.78 \\
\hline 5-Metoxi-luteolinidin $(\mu \mathrm{g})$ & nd & nd & 6.82 & 13.65 \\
\hline 7-Metoxi-apigeninidin $(\mu \mathrm{g})$ & nd & nd & 3.27 & 6.54 \\
\hline $\begin{array}{l}\text { Total proanthocyanins (mg } \\
\text { EC) })^{\mathrm{a}}\end{array}$ & nd & nd & 8.20 & 16.41 \\
\hline $\begin{array}{l}\text { Total phenolic compounds (mg } \\
\qquad \text { AG) }\end{array}$ & nd & nd & 0.08 & 0.16 \\
\hline \multicolumn{5}{|l|}{ Calories (kcal) } \\
\hline Carbohidrates (\%) & 72.58 & 42.42 & 41.09 & 39.82 \\
\hline Protein (\%) & 17.96 & 16.74 & 17.12 & 17.58 \\
\hline Lipids (\%) & 9.47 & 40.85 & 41.79 & 42.61 \\
\hline Caloric density (kcal/g) & 3.80 & 4.66 & 4.56 & 4.44 \\
\hline
\end{tabular}

nd: not determined; BHT: Butylated hydroxytoluene.

Standard diet for rodents (A); High fat diet (B); High fat diet $+50 \%$ substitution of cellulose and $100 \%$ of corn starch by extruded sorghum flour (C); High fat diet $+100 \%$ substitution of cellulose and corn starch for extruded sorghum flour (D).

a Equivalent of catechin.

b Gallic acid equivalent.

( $n=8$ ) as a lean control for 49 days, corresponding to phase I of the study. At the end of this phase, the rats were fasted for $12 \mathrm{~h}$ for collection of blood samples by caudal puncture. Biometric measurements and biochemical analyzes were performed to confirm the induction of obesity and metabolic syndrome in these animals, respectively (data not shown). In phase II, the AIN-93 M group was maintained and the obese HFD group animals were redistributed into three groups according to mean body weight ( $303.8 \mathrm{~g} \pm 1.4 ; \mathrm{n}=8$ /group). The sorghum grain flours of the extruded SC 319 genotype were added to the diets of two of these groups in amounts sufficient to provide 50\% (HFDS 50) and $100 \%$ (HFDS 100) of the recommended dietary fiber $(5 \mathrm{~g}$ ), which replaced $100 \%$ of cornstarch from the diets (Table 1). The other ingredients of the diet (casein, maltodextrin, sucrose and soybean oil) were adjusted to provide isocaloric hyperlipidic diets with or without ESF addition. The weight and feed intake of each animal were monitored weekly for 56 days.

At the end of phase II, after a 12-h fast, the animals were anesthetized with $100 \%$ isoflurane (Isoforine, Cristália ${ }^{\circledR}$ ) and euthanized by cardiac puncture. The blood was collected in appropriate tubes, $16 \times 100 \mathrm{~mm}\left(\mathrm{BD}\right.$ Vacutainer $\left.{ }^{\circledast}\right)$ and centrifuged under refrigeration for $10 \mathrm{~min}$ at $1006 \times g$, to obtain serum. The heart, brain, cecum and liver organs were removed and then weighed. The liver was immediately immersed in liquid nitrogen and subsequently stored at $-80^{\circ} \mathrm{C}$. Some samples of the liver tissue were washed in saline solution, fixed in $10 \%$ formaldehyde and kept at room temperature for further histological analysis.

\subsection{Gene expression levels in hepatic tissue}

RNA total extraction was performed using Trizol Reagent 
(Invitrogen, CA, USA) according to the manufacturer protocols. The RNA concentration and purity was evaluated by Microdrop plate spectrophotometer Multiskan $^{\mathrm{TM}}$ GO (Thermo Scientific, DE, USA), and its integrity was confirmed by electrophoresis agarose gel. A M-MLV Reverse Transcriptase Kit (Invitrogen) was used for cDNA synthesis. The gene expression relative quantification was performed by RT-qPCR using AB StepOne Real Time PCR System equipment and Fast SYBR Green Master Mix (Applied Biosystems, CA, USA) reagent. The initial parameters used to run were $20 \mathrm{~s}$ at $95^{\circ} \mathrm{C}$ and then 40 cycles at $95^{\circ} \mathrm{C}$ ( $3 \mathrm{~s}), 60^{\circ} \mathrm{C}(30 \mathrm{~s})$ followed by melting curve analysis. The primers used on amplification are listed as follows: PPAR- $\alpha$ (Peroxisome proliferatoractivated receptor): CCT GGC TTC CCT GTG AAC T (Forward); ATC TGC TTC AAG TGG GGA GA (Reverse); AdipoR2 (Adiponectin receptor 2): CAT GTT TGC CAC CCC TCA GTA (Forward); ATG CAA GGT AGG GAT GAT TCC A (Reverse); SREBP-1c (Sterol regulatory elementbinding proteins 1c): CGC TAC CGT TCC TCT ATC AAT GAC (Forward); AGT TTC TGG TTG CTG TGC TGT AAG (Reverse); CPT-1 $\alpha$ : GTA AGG CCA CTG ATG AAG GAA GA (Forward); ATT TGG GTC CGA GGT TGA CA (Reverse); and the housekeeping gene GAPDH (Glyceraldehyde-3phosphate dehydrogenase): AGG TTG TCT CCT GTC ACT TC (Forward); CTG TTG CTG TAG CCA TAT TC (Reverse). All primers were designed by using Primer 3 Plus program and obtained from Sigma-Aldrich Brasil Ltda. Gene expression was calculated using 2-Delta-Delta C (T) $\left(2^{-\Delta \Delta \mathrm{Ct}}\right)$ method (Livak \& Schmittgen, 2001), by using GAPDH as reference and obese group as control, which was normalized to 1 .

\subsection{Cytokine and transcription factor}

Serum adiponectin concentrations were analyzed by immunoassay using a commercial ELISA specific kit (Adiponectin Cat. \# EZRADP$62 \mathrm{~K}-$ Millipore ${ }^{\circledast}$, Billerica, MA). Samples were added on microtiter plate and coated with antibody primary monoclonal anti-adiponectin and was incubated for $2 \mathrm{~h}$. Afterwards biotin conjugated antibody Horseradish Peroxidase (HRP) - Avidin was incubated for $1 \mathrm{~h}$. The enzyme-substrate reaction finished with sulfuric acid solution addition and color change was determined spectrophotometrically (Awareness ${ }^{\circledR}$, Stat Fax 2100) in a $450 \mathrm{~nm}$ wavelength. The results were determined by comparing absorbance samples with standard curve.

To determine peroxisome proliferator-activated receptor (PPAR- $\alpha$ ) hepatic concentrations, hepatic tissue samples were homogenized using NE-PER Nuclear and Cytoplasmic Extraction Reagents Kit (Thermo Scientific Fisher) to separate proteins from nuclear fraction and cytoplasmic, according to manufacturer's instructions. Nuclear fraction PPAR- $\alpha$ was assessed by immunoassay using Elisa PPAR-Rat Kit (Cat \# E-EL-R0725-ra; Elabscience, USA), according to manufacturer's recommendations. The microplate that ELISA kit provided was precoated with a PPAR- $\alpha$ antibody specific. Absorbance was measured spectrophotometrically in a $450 \mathrm{~nm}$ wavelength. The PPAR- $\alpha$ concentration on samples was calculated by comparing them with corresponding standard curve.

\subsection{Molecular modeling}

All computer applications were run on OpenSUS Tumbleweed. The ligands: Luteolinidin, Apigeninidin, 5-Methoxy-luteolinidin, 7Methoxy-apigeninidin and Cyanidin (used as standard drug) structures were constructed using Maestro 10.2.010 (Maestro, Version 10.2.010; Schrödinger, LLC, New York, NY, USA). The software LigPrep 3.4 (LigPrep, Version 3.4; Schrödinger, LLC) was used to construct and prepare the ligands involved in this study. The PPAR- $\alpha$ (Protein Data Bank [PDB] ID: 3G8I) (Benardeau et al., 2009) crystallographic structure was obtained from PDB database and Protein Preparation Wizard (Schrödinger, LLC) software was used to prepare this receptor. The OPLS3 force field in the MacroModel 9.9 (MacroModel, Version 9.9; Schrödinger, LLC) was used for optimization. Molecular docking studies between PPAR- $\alpha$ and ligands were performed using Induced Fit
Docking Software (Induced Fit Docking, Version 9.9; Schrödinger, LLC). All computer software belongs to Schrödinger suite.

\subsection{Hepatic samples histological study}

The hepatic tissue samples were fixed in resin. Sections were cut at $3 \mu \mathrm{m}$ thick, mounted on glass slides and stained with hematoxylin and eosin. Glass slides analyzes were performed under a light microscope (Nikon Phase Contrast 0.90 Dry $^{\circledR}$, Japan) and images were captured using a DIGI-PRO 5.0 M digital camera via Micrometrics SE Premium Software (Accu-Scope ${ }^{\circledast}$ ). The histological sections images were captured in a $40 \times$ objective. Fat vesicles, cytoplasm and nucleus were analyzed using Image $\mathrm{J}^{\circledast}$ version 1.5 software (Wayne Rasband). The steatosis degree was evaluated semi quantitatively according to $5^{\circ}$ scale: degree 0 , if fat percentage was absent or $<5 \%$; Grade 1 , if $\geq 5 \%$ and $<25 \%$; Grade 2 , if $\geq 25 \%$ and $<50 \%$; Grade 3 , if $\geq 50 \%$ and $<$ $75 \%$; and grade 4 , if $\geq 75 \%$ (Turlin et al., 2001).

\subsection{Statistical analysis}

Data were analyzed using the Statistical Package for Social Sciences (SPSS) software version 20.0 and expressed as the mean \pm S.D. Statistically significant differences between groups were calculated using variance analysis (ANOVA) followed by the Tukey test. P-values lower than $0.05(\mathrm{p}<0.05)$ were considered statistically significant. The graphs were made using the software Graphpad Prism version 6.0. (GraphPad Software, San Diego, CA).

\section{Results}

\subsection{Effect of diet on dietary intake, body weight, and body mass index}

In general, the dietary intake of the obese control group (HFD) was lower than that of the lean control group (AIN-93 M) and the body weight was higher $(\mathrm{p}<0.05)$ (Fig. $1 \mathrm{~A}$ and $\mathrm{C}$ ). Dietary intake in the HFDS50 and HFDS100 groups was similar to that in the lean control and obese control groups until the seventh week, when there was a higher food consumption by the groups fed ESF ( $p<0.05$ ). Food efficiency was significantly higher $(\mathrm{p}<0.05)$ in groups fed the HFD with or without ESF (Fig. 1B).

ESF consumption did not interfere with the body weight of animals fed a HFD. However, the body mass index was lower in obese animals fed ESF than in the obese control group $(\mathrm{p}<0.05)$ and was similar to that in the lean control group ( $<<0.05$ ) (Fig. 1C and D). Liver weight and the hepatosomatic index were higher in the obese control group (both $\mathrm{p}<0.05$ ) and were decreased in the HFDS50 and HFDS100 groups (both $\mathrm{p}<0.05$ ). The cecum weight and its somatic index was decreased in the obese control group (both $\mathrm{p}<0.05$ ). ESF consumption in the HFDS50 and HFDS100 groups interfered with cecum weight and its somatic index, making them similar to that in the lean control group ( $\mathrm{p}<0.05$ ), although the body weight was not different from that in the obese control group. There was no statistical difference in brain and heart weight between the experimental groups (Fig. 1E and F).

\subsection{Gene expression levels in hepatic tissue and PPAR- $\alpha$ quantification}

The expression of PPAR- $\alpha$ - a nuclear receptor family member that regulates lipid metabolism by mediating fatty acid uptake, activation, and oxidation - decreased in HFD $(\mathrm{p}<0.05)$. ESF treatment increased PPAR- $\alpha$ mRNA expression to 5.56-fold in HFDS50 and 14.25-fold in HFDS100 ( $<<0.05$; Fig. 2A), but the PPAR- $\alpha$ protein hepatic levels increased only in the HFDS100 group ( $<<0.05$; Fig. 2B). Gene expression of the hepatic adiponectin receptor (AdipoR2) was decreased in the HFD group, but was increased 2-fold in the HFDS100 group ( $\mathrm{p}<0.05$; Fig. 2C). No difference was observed between serum 


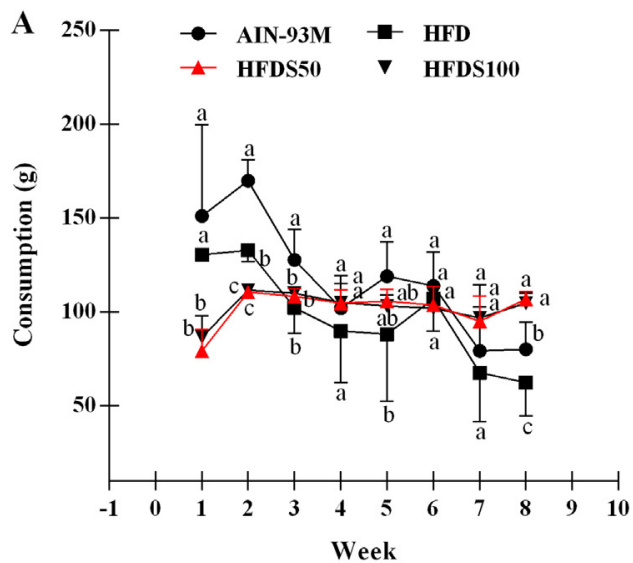

C

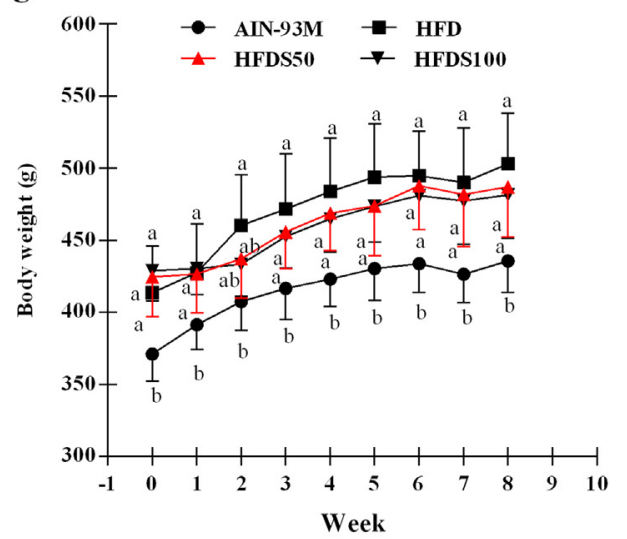

E

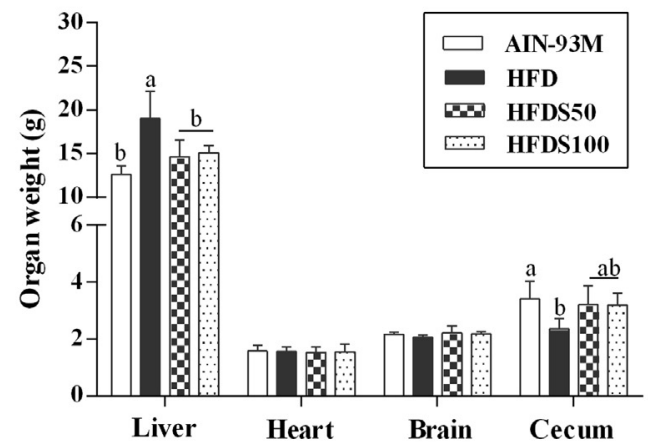

B

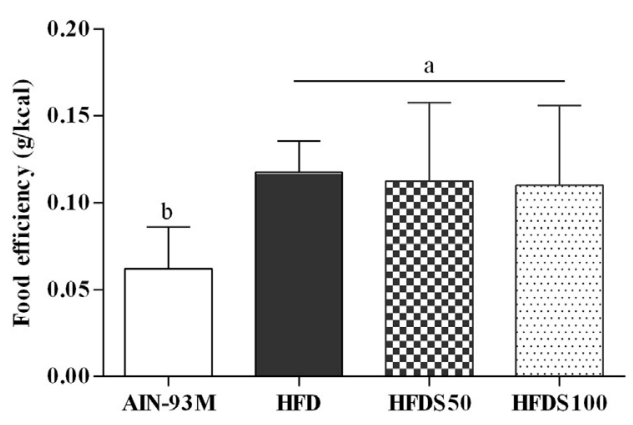

D

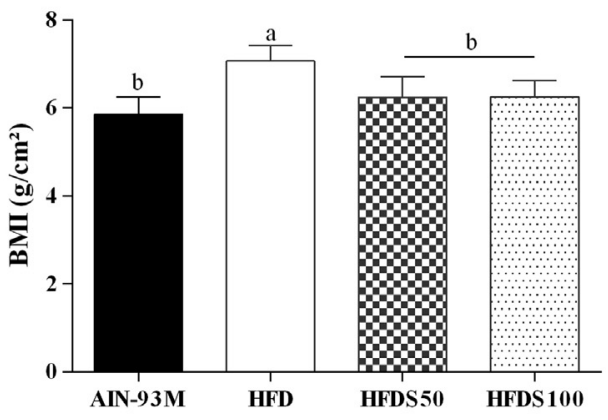

F

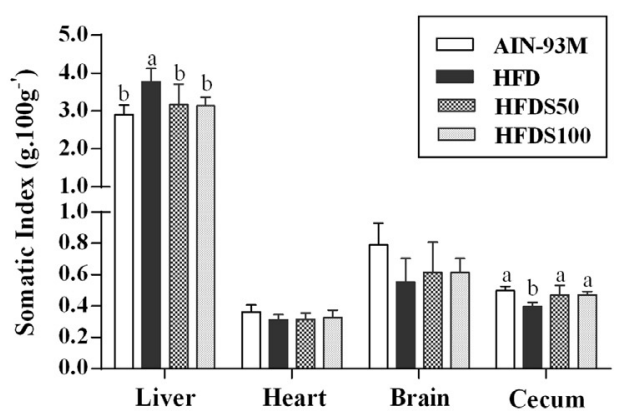

Fig. 1. The food consumption (A), food efficiency (B), body weight (C), Body Mass Index (BMI) (D), organ weight (E) and the liver, heart, brain and cecum somatic index of rats treated with different diets for the 8 weeks (F). Each value is expressed as mean \pm standard deviation. ${ }^{\text {a,b }}$ Different letters indicate statistically significant difference $(p<0.05)$. ANOVA followed by Tukey's test. AIN-93 M: lean control group; HFD: obese control group; HFDS50: test group with extruded sorghum meal replacing $50 \%$ cellulose and $100 \%$ corn starch in a high fat diet; HFDS100: test group with extruded sorghum meal replacing 100\% cellulose and $100 \%$ corn starch in a high fat diet. adiponectin levels in the experimental groups; however, there was a trend to adiponectin hormone reduction in the obese control group $(\mathrm{p}=0.08$; Fig. 2D).

The expression of PPAR- $\alpha$ target genes, such as carnitine palmitoyl transferase (CPT-1 $\alpha$ ), was decreased in animals fed the HFD when compared to AIN-93 M ( $\mathrm{p}<0.05$; Fig. 2E). However, in the HFDS50 and HFDS100 groups, the CPT-1 $\alpha$ mRNA expression did not differ from that in the obese control group ( $p>0.05$ ).

In the obese control group animals, the mRNA expression of sterol regulatory element-binding protein 1c (SREBP-1c) - a nuclear transcription factor that regulates fatty acid synthesis - was significantly increased compared to that in animals from the AIN-93 M group ( $\mathrm{p}<0.05$, Fig. 2F). ESF treatment significantly reduced SREBP-1c expression to 0.26 -fold in HFDS50 ( $\mathrm{p}<0.05$ ) and to 0.13 -fold in HFDS100 ( $\mathrm{p}<0.05)$.

\subsection{Histology of hepatic samples}

Significant changes were observed in cellular components of liver tissue between the AIN-93 M, HFD, HFDS50, and HFDS100 groups during the 8-week treatment (Fig. 3A, B, C and D). The percentage of fat vesicles in the obese control group was higher than in the lean control group and ESF was able to reverse hepatic steatosis in both the HFDS50 and HFDS100 groups ( $<<0.05$; Fig. 3E). The nucleus and cytoplasm percentage in the obese control group was lower than in the lean control group ( $p<0.05)$. ESF consumption increased the cytoplasmic content in the HFDS50 and HFDS100 groups, while the nuclear percentage increased only in the HFDS100 group ( $p<0.05$; Fig. 3E). The lean control group was classified as grade 0 steatosis; HFD consumption in the obese control group increased steatosis to grade 3 and ESF consumption significantly reduced steatosis to grade 2 in both the HFDS50 and HFDS100 groups ( $\mathrm{p}<0.05$, Fig. $3 \mathrm{~F}$ ). 
A

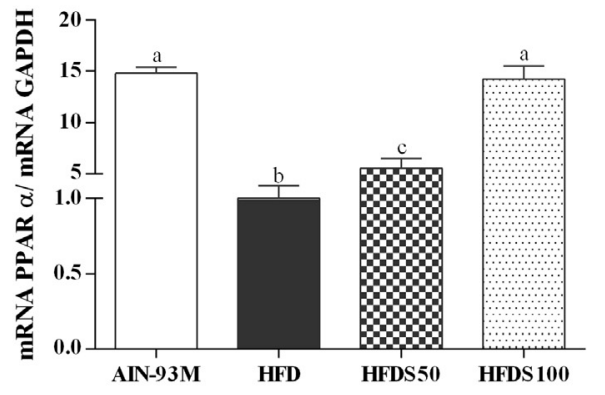

C

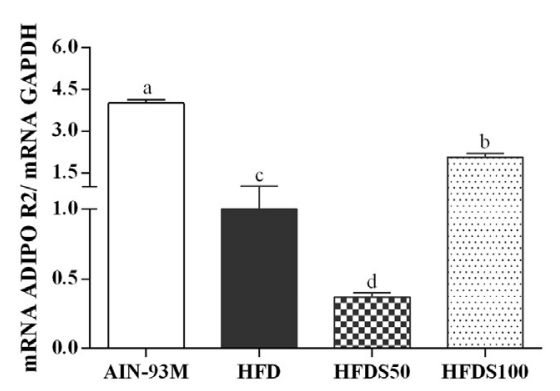

E

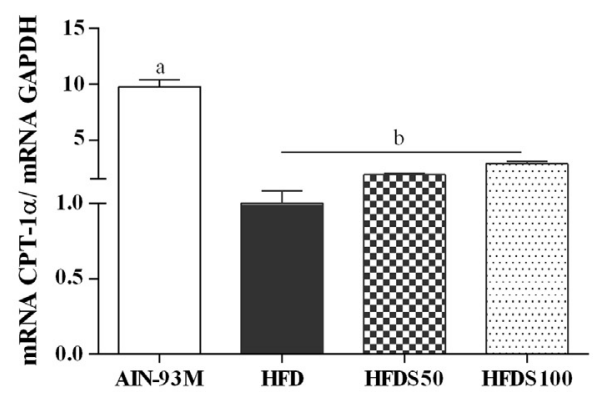

B

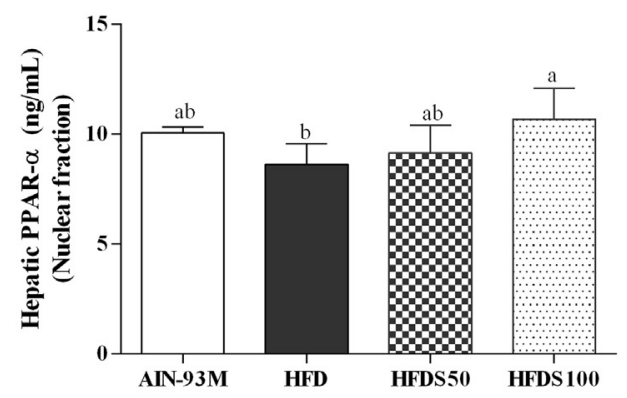

D

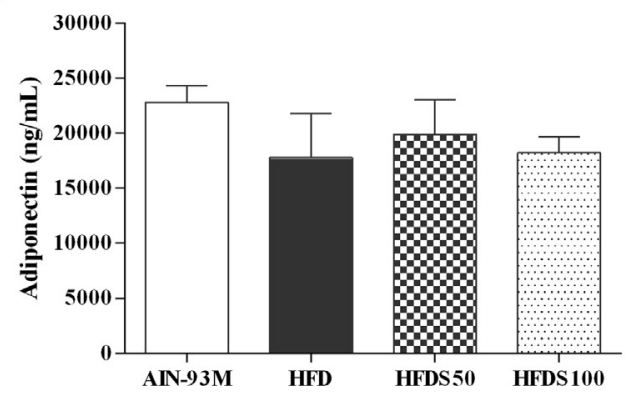

F

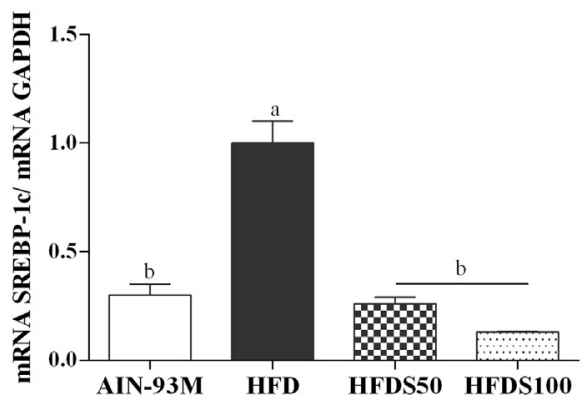

Fig. 2. Adiponectin serum levels $(\mathrm{ng} / \mathrm{mL}$ ), PPAR- $\alpha$ hepatic levels (ng/mL) and genes expression involved in lipolysis and hepatic lipogenesis of obese Wistar rats fed with extruded sorghum flour in a high fat diet for eight weeks. Each value is expressed as mean \pm standard deviation. ${ }^{\text {a,b }}$ Different letters indicate statistically significant difference $(p<0.05)$. ANOVA followed by Tukey's test.

AIN-93 M: lean control group; HFD: obese control group; HFDS50: test group with extruded sorghum meal replacing 50\% cellulose and 100\% corn starch in a high fat diet; HFDS100: test group with extruded sorghum meal replacing $100 \%$ cellulose and $100 \%$ corn starch in a high fat diet.

\subsection{Molecular modeling}

Molecular docking analysis of the 3-deoxyanthocyanidins and their methoxylated forms luteolinidin, apigeninidin, 5-methoxy-luteolinidin, 7-methoxy-apigeninidin, and the standard PPAR- $\alpha$ agonist cyanidin with PPAR- $\alpha$ receptor (Zoete et al., 2007), produced docking score values of $-9.837,-9.676,-9.234, \quad-9.507$, and $-10.345 \mathrm{kcal}_{\mathrm{mol}} \mathrm{m}^{-1}$, respectively (Table 2). The main interactions are shown in Fig. 4, demonstrating that affinity scores can lead to agonist receptor activity. Fig. 4A, B, C, and D depict the molecular docking results of the five ligands in two-dimensional format with PPAR- $\alpha$ amino acids. Fig. 4E, F, G, and H present the best molecular docking from among the evaluated compounds.

\section{Discussion}

The purpose of this study was to investigate the benefit of ESF on the biometric profile and modulation of both lipogenesis and hepatic lipolysis on Wistar rats fed an HFD, because of a high level of bioactive compounds including 3-DXA, proanthocyanins (tannins), and dietary fiber in brown pericarp sorghum (SC 319) (Cardoso et al., 2017). Food intake was generally lower in the obese group compared to the lean control group; possibly due to the high energy density of the HFD. However, the food intake in the HFDS50 and HFDS100 groups was higher than in both the lean and obese controls groups in the last week of the experiment.

Sorghum is a bitter-tasting astringent cereal because of the high content of proanthocyanins (tannins) in its grains. According to Cardoso et al. (2015) the extrusion processing reduces high molecular weight proanthocyanins (oligomers and monomers) into low molecular weight compounds (monomers and dimers), making food palatable. In addition, low molecular weight proanthocyanins are more bioavailable, and can bind to carbohydrates and proteins forming insoluble complexes that are not absorbed, thereby contributing to the meal caloric reduction (Al-Mamary et al., 2001; Cardoso et al., 2015). Thus, lower calorie availability in HFD diets helps to explain a higher dietary intake associated with body weight maintaining and BMI decrease in the HFDS50 and HFDS100 groups.

Our study demonstrates that animals consuming HFD lower cecum weights and somatic index, compared to animals consuming ESF. On the other hand, there was an increase in total liver weight in animals consuming the HFD and a reduction in animals consuming ESF. Lyons, Kennedy, and Roche (2016) also observed that the consumption of HFD contributes to accumulation of fat in tissues such as the liver, heart, pancreas, and muscle, leading to lipotoxicity and contributing to the development of chronic non-communicable diseases.

The increased liver weight in animals consuming HFD can be explained by the higher fat accumulation in this tissue (three degrees of 

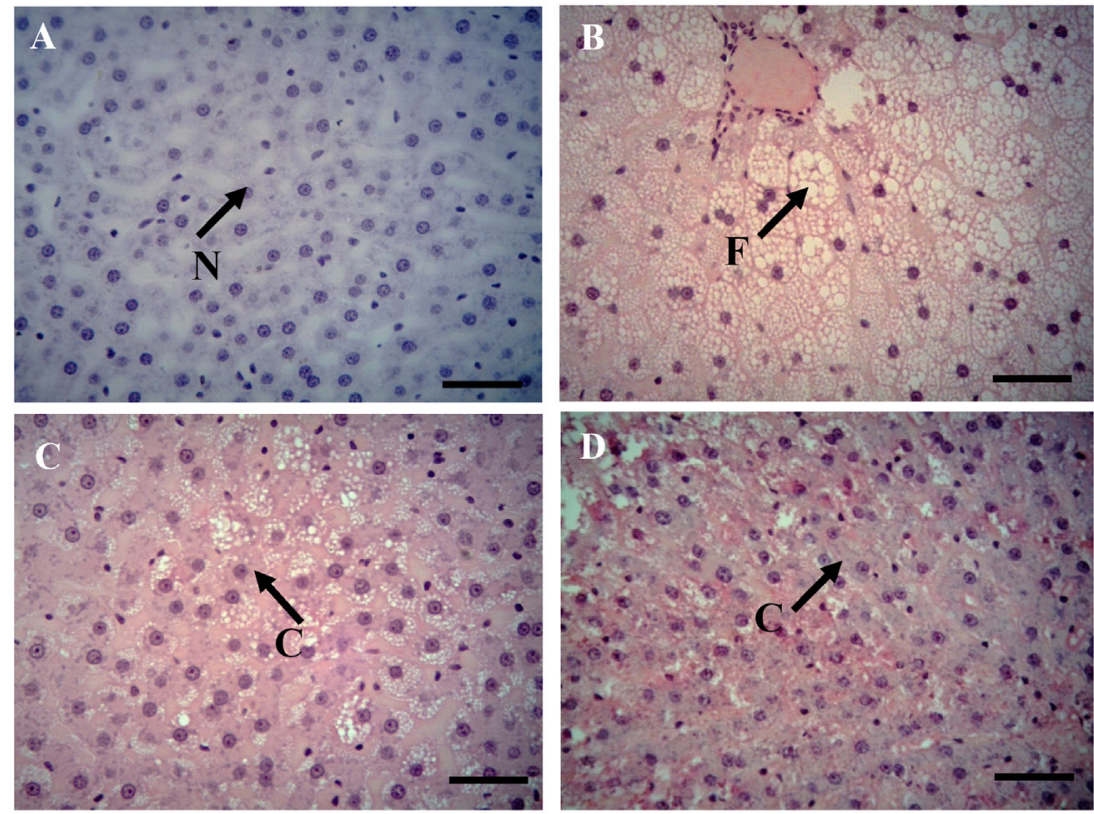

Fig. 3. Hepatic tissue phenotype (A: AIN93M; B: HFD; C: HFDS50; D: HFDS100), cellular components percentage (E) and steatosis degree (F) of obese Wistar rats fed with extruded sorghum flour in a high fat diet for eight weeks. Each value is expressed as mean \pm standard deviation. a,b Different letters indicate statistically significant difference $(p<0.05)$. ANOVA followed by Tukey's test.

AIN-93 M: lean control group; HFD: obese control group; HFDS50: test group with extruded sorghum meal replacing 50\% cellulose and $100 \%$ corn starch in a high fat diet; HFDS100: test group with extruded sorghum meal replacing $100 \%$ cellulose and $100 \%$ corn starch in a high fat diet.

N: Nucleus; F: Fat vesicles; C: Cytoplasm. HE staining. Barr: $20 \mu \mathrm{m}$.

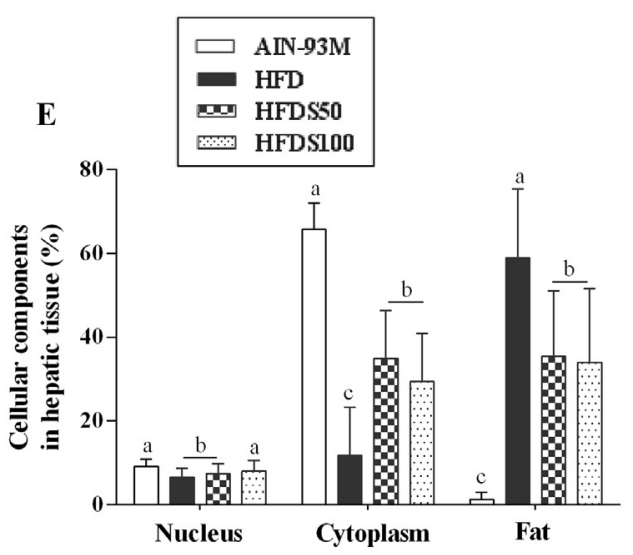

Table 2

Values of Glide Score (GScore), the number of interaction by Hydrogen bonds (Hbond) and by van der Waals (good $v d W$ ) between the ligands and PPAR- $\alpha$ receptor (Schrödinger Suite, Induced Fit Docking Program).

\begin{tabular}{llll}
\hline Ligand & GScore $\left(\mathrm{kcal} \mathrm{mol}^{-1}\right)$ & Hbond & Good vdW \\
\hline Luteolinidin & -9.837 & 5 & 164 \\
Apigeninidin & -9.676 & 3 & 230 \\
7-Methoxy-Apigeninidin & -9.507 & 1 & 251 \\
5-Methoxy-Luteolinidin & -9.234 & 2 & 288 \\
Cyanidin (standard) & -10.345 & 5 & 220 \\
\hline
\end{tabular}

steatosis). In addition, livers from animals on HFD presented a lower percentage of cellular components including cytoplasm and nuclei, when compared to the livers from animals in the lean control group. In contrast, animals consuming ESF displayed a decreased degree of steatosis from 3 to 2, reflecting a reduction in body weight and somatic index, as well as increases in the percentages of nuclei and cytoplasm. These results show that, despite the apoptosis evident in hepatic cells from obese animals fed HFD, ESF was able to reverse the hepatic injury caused by lipotoxicity. The same effects were observed in rats following the consumption of white bread with added bioactive compounds, and fed an HFD (Pozzo et al., 2015). Therefore, bioactive compounds, such as tannins, anthocyanins, and phenolic acids, as well as the dietary fiber present in extruded brown sorghum may explain the results in our study, though precise mechanisms of action cannot be identified in this study (Cruz et al., 2016; Kieffer, Martin, \& Adams, 2016).

The liver is the main organ for lipogenesis. Therefore, increased circulation of free fatty acids in an HFD can activate SREBP-1c in the liver. SREBP-1c is a transcription factor that is sensitive to nutritional and hormonal regulation, which controls the expression of a variety of enzymes necessary in triacylglycerol synthesis and storage (Jeon \& Osborne, 2012). Presently, the HFD increased the SREBP-1c mRNA expression, indicating a lipogenic process. ESF addition to HFD reduced the expression of SREBP-1c mRNA regardless of the amount added. This may be associated with the reduced hepatic steatosis in these animals. These findings suggest that ESF plays an important role in endogenous fatty acid synthesis control, lipid accumulation, and lipotoxicity.

On the other hand, PPAR- $\alpha$ regulates the expression of genes involved in the pathways of peroxisomal and mitochondrial $\beta$-oxidation as well as fatty acid transport proteins such as CPT-1, which plays an important role in fatty acid oxidation and reduction of triacylglycerol (Mello, Materozzi, \& Galli, 2016). Obese rats fed an HFD tend to develop hypoadiponectinemia associated with lower expression of peripheral adiponectin receptors (AdipoR1 and AdipoR2) and reduced activity of energy-critical sensors such as PPAR- $\alpha$ and target genes such as CPT-1 (Yamauchi et al., 2007).

Adiponectin activity is important for PPAR- $\alpha$ activation as observed in this study, although the serum levels did not differ significantly between the groups studied. Animals fed with $100 \%$ ESF dietary fiber 

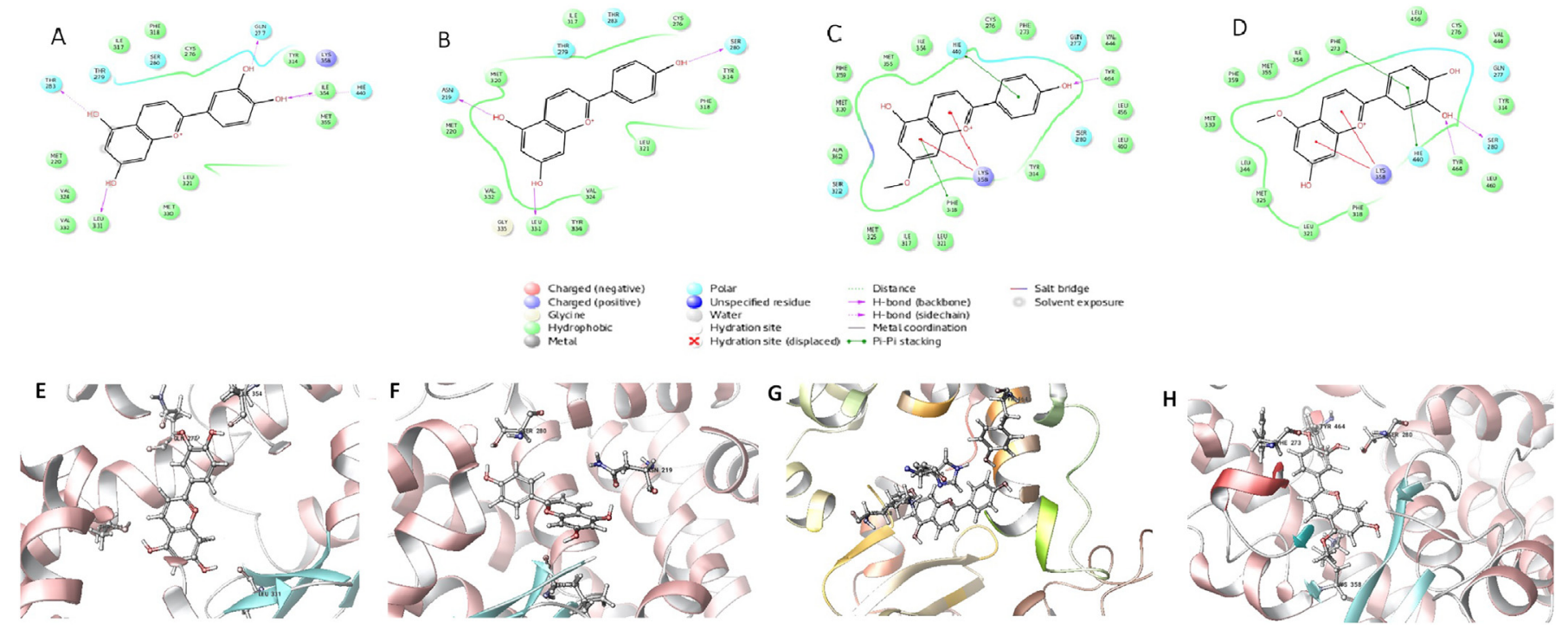

Fig. 4. Interactions between amino acids of PPAR- $\alpha$ receptor site and Luteolinidin (A), Apigeninidin (B), 7-Methoxy-apigeninidin (C) and 5-Methoxy-luteolinidin (D) and representation of molecular docking results between Luteolinidin (E), Apigeninidin (F), 7-Methoxy-apigeninidin (G) and 5-Methoxy-luteolinidin (H) and PPAR- $\alpha$ receptor site.

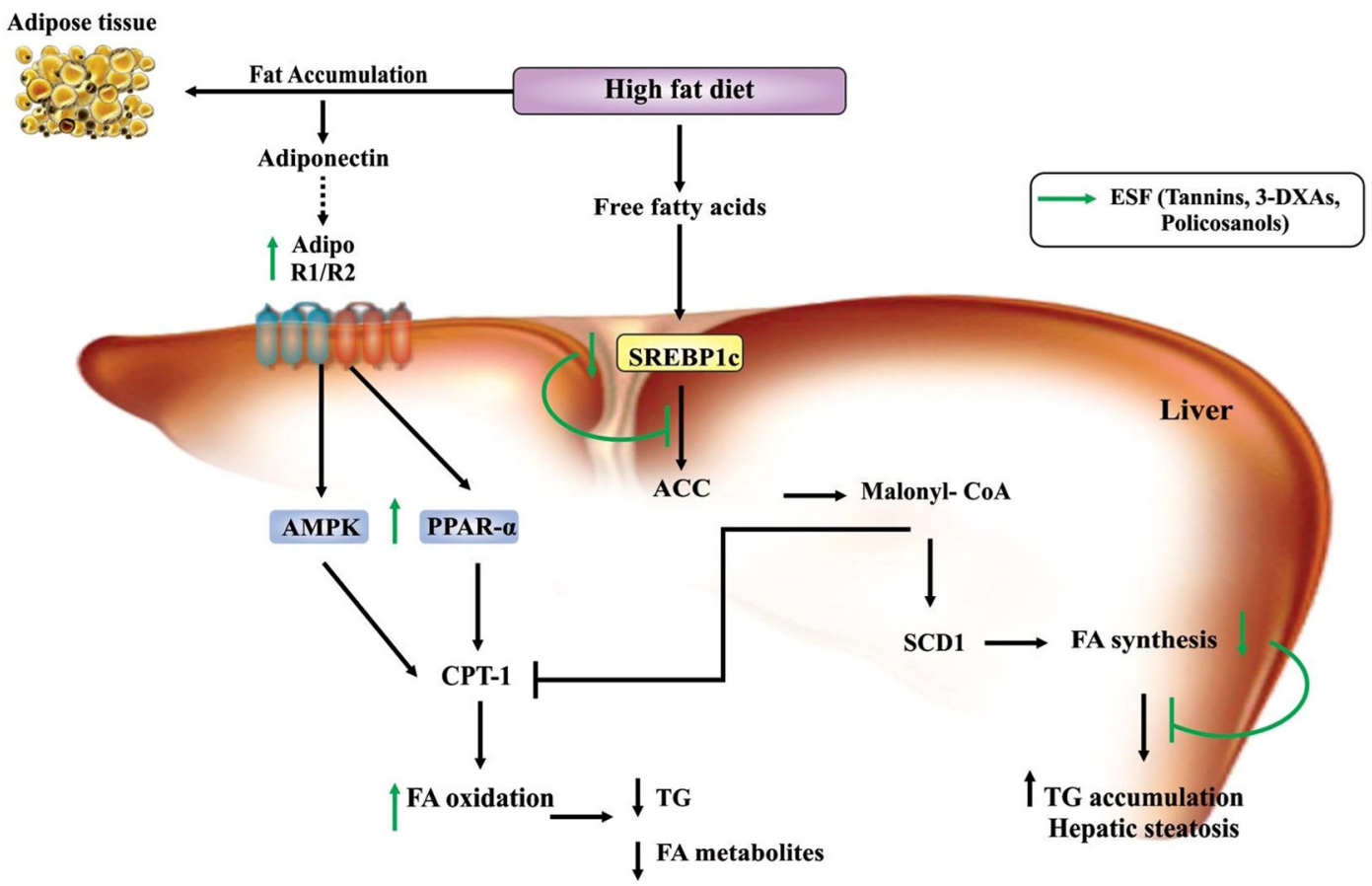

Fig. 5. Potential action mechanism of bioactive compounds present in brown pericarp extruded sorghum flour in the lipogenic pathway and fatty acids lipolysis in the hepatic tissue of obese Wistar rats.

ACC: Acetyl-CoA carboxylase; AMPK: AMP-activated protein kinase; Adipo R1/R2: Adiponectin receptor 1/2; CPT1: Carnitine palmitoyltransferase 1; FA: Fatty acid; FAS: Fatty acid synthase; PPAR- $\alpha$ : Peroxisome proliferator-activated receptor $\alpha$; SCD1: Stearoyl-CoA desaturase; SREBP1: Sterol regulatory element-binding proteins; ESF: extruded sorghum flour; 3-DXAs: 3-deoxyanthocyanidins. (For interpretation of the references to color in this figure legend, the reader is referred to the web version of this article.)

displayed increased mRNA expression of the hepatic AdipoR2 receptor and increased expression and activity of PPAR- $\alpha$, but without changes in the expression of target genes (CPT-1). However, animals fed $50 \%$ ESF dietary fiber showed reduced AdipoR2 receptor and, although there was an increase in the expression of PPAR- $\alpha$ mRNA, the protein levels remained unchanged. The increase in hepatic PPAR- $\alpha$ expression in animals treated with ESF by replacing $100 \%$ dietary fiber may be explained based on the compounds present in the brown pericarp sorghum; 3-DXA (luteolinidine, apigeninidine, 7-methoxy-apigeninidine, and 5-methoxy-luteolinidine) was consumed in large quantities by this group of animals.

Isolated anthocyanins or their extracts can bind directly to PPAR- $\alpha$ and act as agonists favoring both increased expression and activity of this receptor (Jia et al., 2013; Seymour et al., 2008; Tsuda et al., 2003; Yang et al., 2011). The present molecular plating analysis revealed good affinity values (Gscore) between PPAR- $\alpha$ and luteolinidine, apigeninidine, 7-methoxy-apigeninidine, 5-methoxy-luteolinidine, and standard cyanidin agonist. Luteolinidine displayed the best affinity 
value (GScore $-9837 \mathrm{kcal}_{\mathrm{mol}}{ }^{-1}$ ) and was comparable to the known PPAR- $\alpha$ agonist, cyanidin (GScore $-10,345 \mathrm{kcal}^{\mathrm{mol}}{ }^{-1}$ ). In addition, apigenidine, 7-methoxy-apigeninidine, and 5-methoxy-luteolinidine showed good affinity values and hydrogen bonding with amino acid residues. These results suggest an interaction that may lead to agonist activity of the 3-deoxyanthocyanidins and their methoxylated forms present in the EFS to the PPAR- $\alpha$ receptor, which may favor the regulation of hepatic lipolysis.

The beneficial effects observed after EFS consumption show that sorghum extrusion improved biometric markers and hepatic steatosis by regulation of transcription factors of the lipogenic pathway. EFS contributed to reduced hepatic steatosis by inhibiting fatty acids synthesis and decreasing the SREBP-1c expression, possibly blocking the production of lipogenic enzymes including acetyl coenzyme A carboxylase, fatty acid synthase, and estearoil coenzyme A desaturase 1 , while promoting increased $\beta$-oxidation by the increased sensitization of the AdipoR2 receptor and increased PPAR- $\alpha$ (Fig. 5).

\section{Conclusion}

Consumption of ESF, which is rich in bioactive compounds, promotes hepatic lipogenesis control by reducing fat accumulation in the liver of animals fed an HFD. This contributes to improvements in biometric measurements in obese Wistar rats. ESF has potential value as a component of foods to assist in the prevention of obesity and hepatic steatosis.

\section{Conflicts of interest}

The authors declare no conflict of interest.

\section{Acknowledgments}

The authors would like to thank the Brazilian funding agencies: Empresa Brasileira de Pesquisa Agropecuária Milho e Sorgo (EMBRAPA grant number: 13/2014), Conselho Nacional de Desenvolvimento Científico e Tecnológico (CNPq grant number: 305655/2013-2), Rede Mineira de Química (RQ-MG) supported by FAPEMIG (CEX - RED00010-14) and INCT-INOFAR (grant CNPq 573.564/2008-6).

\section{References}

Alam, M. S., et al. (2016). Extrusion and extruded products: Changes in quality attributes as affected by extrusion process parameters: A review. Critical Review in Food Science and Nutrition, 56, 445-475.

Al-Mamary, M., et al. (2001). In vivo effects of dietary sorghum tannins on rabbit digestive enzymes and mineral absorption. Nutrition Research, 21, 1393-1401.

Anunciação, P. C., et al. (2017). Comparing sorghum and wheat whole grain breakfast cereals: Sensorial acceptance and bioactive compound content. Food Chemistry, 221, 984-989.

Arbex, P. M., et al. (2018). Extruded sorghum flour (Sorghum bicolor L.) modulate adiposity and inflammation in high fat diet-induced obese rats. Journal of Functional Foods, 42, 346-355.

Belobrajdic, D. P., et al. (2011). Cereal based diets modulate some markers of oxidative stress and inflammation in lean and obese Zucker rats. Nutrition \& Metabolism, 8, $27-37$.

Bénardeau, A., et al. (2009). Aleglitazar, a new, potent, and balanced dual PPAR $/ \gamma$ agonist for the treatment of type II diabetes. Bioorganic \& Medicinal Chemistry Letters, $19,2468-2473$.

Cardoso, L. D. M., et al. (2015). Phenolic compounds profile in sorghum processed by extrusion cooking and dry heat in a conventional oven. Journal of Cereal Science, 65,
$220-226$.

Cardoso, L. D. M., et al. (2017). Sorghum (Sorghum bicolor 1.): Nutrients, bioactive compounds, and potential impact on human health. Critical Reviews in Food Science and Nutrition, 57, 372-390.

Ceriello, A., \& Motz, E. (2004). Is oxidative stress the pathogenic mechanism underlying insulin resistance, diabetes, and cardiovascular disease? The common soil hypothesis revisited. Arteriosclerosis, Thrombosis and Vascular Biology, 24, 816-823.

Chung, I.-M., et al. (2011). Antidiabetic effects of three Korean sorghum phenolic extracts in normal and streptozotocin-induced diabetic rats. Food Research International, 44, 127-132.

Cruz, A. B., et al. (2016). Assessment of bioactive metabolites and hypolipidemic effect of polyphenolic-rich red cabbage extract. Pharmaceutical Biology, 54, 3033-3039.

Day, C. P., \& James, O. F. (1998). Steatohepatitis: A tale of two "hits". Gastroenterology, $114,842-845$.

Dowman, J. K., Tomlinson, J. W., \& Newsome, P. N. (2010). Pathogenesis of non- alcoholic fatty liver disease. Quarterly Journal of Medicine, 103, 71-83.

Goto, T., et al. (2011). Tiliroside, a glycosidic flavonoid, ameliorates obesity-induced metabolic disorders via activation of adiponectin signaling followed by enhancement of fatty acid oxidation in liver and skeletal muscle in obese-diabetic mice. Journal of Nutritional Biochemistry, 23, 768-776.

Jeon, T.-I., \& Osborne, T. F. (2012). SREBPs: Metabolic integrators in physiology and metabolism. Trends in Endocrinology and Metabolism, 23, 65-72.

Jia, Y., et al. (2013). Cyanidin is an agonistic ligand for peroxisome proliferator-activated receptor-alpha reducing hepatic lipid. Biochemical et Biophysica Acta, 1831, 698-708.

Kieffer, D. A., Martin, R. J., \& Adams, S. H. (2016). Impact of dietary fibers on nutrient management and detoxification organs: Gut, liver, and kidneys. Advances in Nutrition, 7, 1111-1121.

Livak, K. J., \& Schmittgen, T. D. (2001). Analysis of relative gene expression data using real-time quantitative PCR and the 2-DeltaCT method. Methods, 25, 402-408.

Lopes, R. D. C. S. O., et al. (2018). Evaluation of the health benefits of consumption of extruded tannin sorghum with unfermented probiotic milk in individuals with chronic kidney disease. Food Research International, 107, 629-638.

Lopez, N. J. S., et al. (2016). The extrusion process as an alternative for improving the biological potential of Sorghum bran: Phenolic compounds and antiradical and antiinflammatory capacity. Evidence-Based Complementary and Alternative Medicine, 8.

Lyons, C. L., Kennedy, E. B., \& Roche, H. M. (2016). Metabolic inflammation-differential modulation by dietary constituents. Nutrients, 8, 247 .

Mello, T., Materozzi, M., \& Galli, A. (2016). PPARs and mitochondrial metabolism: From NAFLD to HCC. PPAR Research18.

Pozzo, L., et al. (2015). Effect of white wheat bread and white wheat bread added with bioactive compounds on hypercholesterolemic and steatotic mice fed a high fat diet Journal of the Science of Food and Agriculture, 95, 2454-2461.

Reeves, P. G., Nielsen, F. H., \& Fahey, G. C. (1993). AIN-93 purified diets for laboratory rodents: Final report of the American Institute of Nutrition ad hoc writing committee on the reformulation of the AIN-76A rodent diet. Journal of Nutrition, 123, 1939-1951.

Seymour, E. M., et al. (2008). Altered hyperlipidemia, hepatic steatosis, and hepatic peroxisome proliferator-activated receptors in rats with intake of tart cherry. Journal of Medicinal Food, 11, 252-259.

Stefoska-Needham, A., et al. (2015). Sorghum: An underutilized cereal whole grain with the potential to assist in the prevention of chronic disease. Food Reviews International, 31, 401-437.

Szczepaniak, L. S., et al. (2005). Magnetic resonance spectroscopy to measure hepatic triglyceride content: Prevalence of hepatic steatosis in the general population. American Journal of Physiology - Endocrinology and Metabolism, 288, 462-468.

Tsuda, T., et al. (2003). Dietary cyanidin 3-O-beta-D-glucoside-rich purple corn color prevents obesity and ameliorates hyperglycemia in mice. Journal of Nutrition, 133, $2125-2130$.

Turlin, B., et al. (2001). Histologic features of the liver in insulin resistance-associated Iron overload. A study of 139 patients. American Journal of Clinical Pathology, 116, 263-270.

Vargas-Solorzano, J. W., et al. (2014). Physicochemical properties of expanded extrudates from colored sorghum genotypes. Food Research International, 55, 37-44.

Yamauchi, T., et al. (2007). Targeted disruption of AdipoR1 and AdipoR2 causes abrogation of adiponectin binding and metabolic actions. Nature Medicine, 13, 332-339.

Yamazaki, T., et al. (2007). Fish oil prevents sucrose-induced fatty liver but exacerbates high-safflower oil-induced fatty liver in ddY mice. Hepatology, 46, 1779-1790.

Yang, Y., et al. (2011). Anthocyanin extract from black Rice significantly ameliorates platelet hyperactivity and hypertriglyceridemia in Dyslipidemic rats induced by high fat diets. Journal of Agricultural and Food Chemistry, 59, 6759-6764.

Zoete, V., Grosdidier, A., \& Michielin, O. (2007). Peroxisome proliferator-activated receptor structures: ligand specificity, molecular switch and interactions with regulators. Biochimica et Biophysica Acta, 1771, 915-925. 